\title{
Regorafenib therapy in metastatic colorectal cancer patients: markers and outcome in an actual clinical setting
}

\author{
M. UNSELD ${ }^{1}$, M. FILIP ${ }^{1}$, S. SEIRL ${ }^{1}$, A. GLEISS ${ }^{2}$, D. BIANCONI ${ }^{1}$, M. KIELER ${ }^{1}$, S. DEMYANETS ${ }^{3}$, W. SCHEITHAUER ${ }^{1}$, C. ZIELINSKI ${ }^{1}$, G. PRAGER ${ }^{1, *}$ \\ ${ }^{1}$ Department of Medicine I, Clin. Div. of Oncology and Comprehensive Cancer Center, Medical University of Vienna, Austria; ${ }^{2}$ Center for \\ Medical Statistics, Informatics, and Intelligent Systems, Medical University of Vienna, Austria; ${ }^{3}$ Department of Laboratory Medicine, Medical \\ University of Vienna, Austria
}

*Correspondence: gerald.prager@meduniwien.ac.at

Received July 27, 2017 / Accepted October 11, 2017

\begin{abstract}
The oral multikinase inhibitor regorafenib had beneficial effects in randomized clinical phase III trials compared to the placebo in patients with metastatic colorectal cancer (mCRC) who progressed on standard therapies. The factors which influence regorafenib response and therapy sequence during treatment history are still highly discussed, and herein we analyzed the therapy algorithm, outcome and clinical markers following regorafenib application in a single center register study. Clinical data for 48 metastatic colorectal cancer patients were collected from 01.01.2013 to 31.12.2016. Treatment effects according to various patient and tumor characteristics were evaluated using univariate and multivariate Cox proportional hazard regression models. The 48 patients comprised 14 (29\%) females and 34 (71\%) males, with mean age $64.2 \pm 9$ and ECOG 0-1. Progression free survival under regorafenib therapy was 2.9 months (quartiles 2.2; 4.4) and the overall response rate was $2(4 \%)$ and disease control rate was 19 (40\%). Overall survival (OS) and progression free survival (PFS) were investigated under regorafenib in the chemotherapy regimen given immediately before and afterthis treatment. Variables including tumor localization, Ras status, CEA and CA 19-9 plasma levels were analyzed for their impact on PFS, and the regorafenib-related adverse events were also observed. Our study confirms the efficacy of regorafenib in a real-life setting. We established that response rate and PFS in regorafenib treatment are independent of tumor localization, Ras status or biomarkers such as CEA and CA 19-9. Trifluridin/tripacil application or re-induction of chemotherapy $+/-$ target therapy was effective following regorafenib therapy.
\end{abstract}

Key words: colon cancer, chemotherapy, regorafenib

In 2012, colorectal cancer was the third most common type of cancer, with 1.4 million new globally diagnosed cases examined in that year [1]. Depending on tumor stage, the 5 -year survival rate decreases from $90 \%$ in non-metastatic patients to just $13 \%$ in patients with diagnosed metastatic colorectal cancer (mCRC) [2].

While the chemotherapeutic options in first and second line treatment of $\mathrm{mCRC}$ patients have been thoroughly investigated, interest in later treatment lines is steadily increasing [3]. Standard regimes in first-line setting include chemotherapeutical drugs such as 5-Fluouracil, oxaliplatin and irinotecan, and these are often combined with targeted therapy (antiVEGF/antiEGFR) [3].

Although treatment efficacy could be significantly improved, further therapy options after standard therapy failure are limited. Regorafenib is an oral multikinase inhib- itor which targets signaling pathways involved in carcinogenesis (KIT, RET, BRAF), angiogenesis (Tie2, VEGFR1-3) and the tumor-micro-environment (PDGFR, FGFR) [4-6].

In 2012, the FDA approved the application of regorafenib based on the results of the international multicenter phase III CORRECT trial [4]. The study met its primary endpoint with significant improvement in OS (6.4 months vs 5.0 months, HR $0.77,95 \%$ CI 0.64 to $0.94, p=0.0052)$. Results were then confirmed in an Asian population in the CONCUR study [7], and further indications for regorafenib were investigated and these led to its application in advanced GIST after progression on imatinib or sunitinib in the GRID trial (PFS 4.8 months for regorafenib vs 0.9 months for placebo [HR] 0.27 , 95\% CI 0.19-0.39; p<0.0001) [5, 8]. Regorafenib has recently become approved for patients with hepatocellular carcinoma who progressed on sorafenib treatment [9]. 
Retrospective data on regorafenib real-life use seeks to address therapy response and cost effectiveness, but little data is available [10-12]. In this single center registry study, we analyzed treatment response after regorafenib administration in different therapy lines and compared clinical outcomes with those achieved in previous studies.

\section{Patients and methods}

Study design. The study was conducted in convention with the International Conference on Harmonization E6 requirements for Good Clinical Practice outlined in the Declaration of Helsinki and approved by the ethics committee of the Medical University of Vienna (EC Nr.:1302/2016).

Patients. Eligible patients had histologically confirmed diagnosis of metastatic colorectal cancer with good performance status (ECOG 0-1), and verified tumor progression after conventional first, second or third line treatment. Measurable disease according to RECIST 1.1 criteria was required and therapy was only given after informed consent and screening of all patients who received regorafenib at the Departement of Oncology at the Medical University of Vienna from January 2013 to December 2016. Other baseline eligibility criteria included calculated creatinine clearance of $>60 \mathrm{~mL} / \mathrm{min}$; adequate bone marrow function indicated by leukocyte count $\geq 3,000 / \mu \mathrm{L}$, absolute neutrophil count $\geq 1,000 / \mu \mathrm{L}$ and platelet count $\geq 100,000 / \mu \mathrm{L}$ and adequate hepatic function with total bilirubin up to $1.5 \times$ the upper reference range.

Treatment plan and toxicity assessment. Regorafenib was prescribed for patients suffering from metastatic colorectal cancer. Dosage reduction was instituted on occurrence of intolerable grade 2 and grade 3 and higher adverse events (AE). Therapy was administered until disease progression, severe AE2 or patient withdrawal from treatment.

Table 1. Descriptive characteristics of mCRC patients.

\begin{tabular}{lcc}
\hline Patients & n & \% \\
\hline Women & 14 & 29 \\
Men & 34 & 71 \\
Age & years & stdev \\
mean & 64.2 & 8.7 \\
Localisation & 48 & 100 \\
right & 13 & 27 \\
left & 35 & 73 \\
TNM: stage IV & 48 & 100 \\
Metastasis & 48 & 100 \\
Lung & 34 & 71 \\
Liver & 37 & 77 \\
Lymphnode & 32 & 67 \\
others & 13 & 27 \\
Surgery & 39 & 81 \\
Radiation & 23 & 48 \\
\hline
\end{tabular}

Toxicities were graded by the National Cancer Institute Common Terminology Criteria for AE version 4.0. The following adverse reactions were evaluated during different therapy lines; vertigo/nausea, fatigue, diarrhea, hypertension, hand-foot-skin reaction, mucositis/stomatitis, weight loss/anorexia and hoarseness

Statistical considerations. The distribution of categorical variables is described by counts and percentages. Age is described by mean and standard deviation. PFS is defined as time from treatment commencement to progression according to RECIST 1.1 criteria, death from any cause or the end of treatment due to side effects. Patients were evaluated at the last-seen date if therapy was ongoing. Overall survival was defined from the date of diagnosis to death from any cause or the evaluation date. Median OS and PFS and quartiles are deduced from Kaplan-Meier estimates shown with $95 \%$ confidence. Hazard ratios for quantifying the potential influence of predictors are calculated by Cox proportional-hazards regression models with adjustment for the log of time from first diagnosis to treatment commencement. CEA and CA_19-9 were transformed by binary logarithm for predictors in a way that their hazard ratio quantified the effect of doubling the respective predictor value. The reported $p$-values are the results of two-sided tests, with p-values $<0.05$ statistically significant. Due to the exploratory character of the study, no correction for multiplicity was performed, and p-values and confidence intervals are to be interpreted accordingly. All computations were by SAS software Version 9.4 (SAS Institute Inc., Cary, NC, USA, 2012).

\section{Results}

Patient characteristics. In this single center analysis, 14 women (29\%) and 34 men (71\%) were identified between 2013 and 2016 as suitable candidates for our database (Table 1). The patients' mean age was $64.2 \pm 8.7$ years and all had an ECOG 0-1 performance score at time of regorafenib therapy. From this 48 patient cohort, 35 (73\%) had left side tumors and 13 (27\%) had right sided tumors, including 3 in the transverse colon. Further, 21 patients (44\%) exhibited Ras mutation, 25 (52\%) were wild type (wt) and the Ras status was unknown in the remaining $2(4 \%)$. All patients suffered from metastatic disease including in the liver (77\%), lung (71\%) and lymph nodes (67\%).

Response/clinical efficacy. The median progression free survival (PFS) under the therapy given immediately before regorafenib administration was 3.1 months (quartiles 2.0; 6.0; Figure 1A). Table 2 shows that 17 (35\%) patients achieved stable disease during their treatment period, while 31 (65\%) were progressive according to RECIST criteria. This gave $35 \%(n=17)$ disease control rate (DCR).

PFS during regorafenib monotherapy was 2.9 months (quartiles 2.2; 4.4; Figure 1B, Table 3), and partial remission was achieved in 2 patients (4\%). The calculated DCR for 
Table 2. Progression free survival (PFS) and tumor response in patients treated before regorafenib.

\begin{tabular}{lcc}
\hline Th before & $\mathbf{n}$ & \% \\
\hline Regorafenib & 48 & 100 \\
ECOG & 48 & 100 \\
0 & 25 & 52 \\
1 & 23 & 48 \\
PFS & median & quartiles \\
months & 3.1 & $2.0 ; 6.0$ \\
response & $\mathrm{n}$ & $\%$ \\
CR & 0 & 0 \\
PR & 0 & 0 \\
SD & 17 & 35 \\
PD & 31 & 65 \\
ORR & 0 & 0 \\
DCR & 17 & 35 \\
\hline
\end{tabular}

Table 3. Progression free survival (PFS), tumor response and adverse events observed during regorafenib treatment.

\begin{tabular}{|c|c|c|}
\hline Th & n & $\%$ \\
\hline Regorafenib & 48 & 100 \\
\hline ECOG & 48 & 100 \\
\hline 0 & 32 & 67 \\
\hline 1 & 16 & 33 \\
\hline PFS & median & quartiles \\
\hline months & 2.9 & $2.2 ; 4.4$ \\
\hline response & $\mathrm{n}$ & $\%$ \\
\hline CR & 0 & 0 \\
\hline $\mathrm{PR}$ & 2 & 5 \\
\hline SD & 17 & 45 \\
\hline $\mathrm{PD}$ & 19 & 50 \\
\hline ORR & 2 & 5 \\
\hline DCR & 19 & 40 \\
\hline \multicolumn{3}{|l|}{ tumormarker } \\
\hline median (quartiles) & before TH & after Th \\
\hline CEA $\mu \mathrm{g} / \mathrm{L}$ & $47(13 ; 294)$ & $90(23 ; 448)$ \\
\hline CA $19-9$ kU/L & $72(19 ; 341)$ & $106(32 ; 106)$ \\
\hline adverse events & $\mathrm{n}(\%)$ & $\mathrm{n}(\%)$ \\
\hline grade & any & III \\
\hline vertigo/nause & $11(23)$ & $0(0)$ \\
\hline fatigue & $24(50)$ & $1(2)$ \\
\hline diarrhea & $11(23)$ & $1(2)$ \\
\hline hypertension & $10(21)$ & $0(0)$ \\
\hline HFS-reaction & $21(44)$ & $6(13)$ \\
\hline mucositis/stomatitis & $10(21)$ & $1(2)$ \\
\hline weight loss & $13(27)$ & $0(0)$ \\
\hline hoarseness & $19(40)$ & $0(0)$ \\
\hline
\end{tabular}

regorafenib therapy was $40 \%$ (19). One patient still received regorafenib at the date of analysis and one patient was lost during follow up (Table 4). No statistical significance was
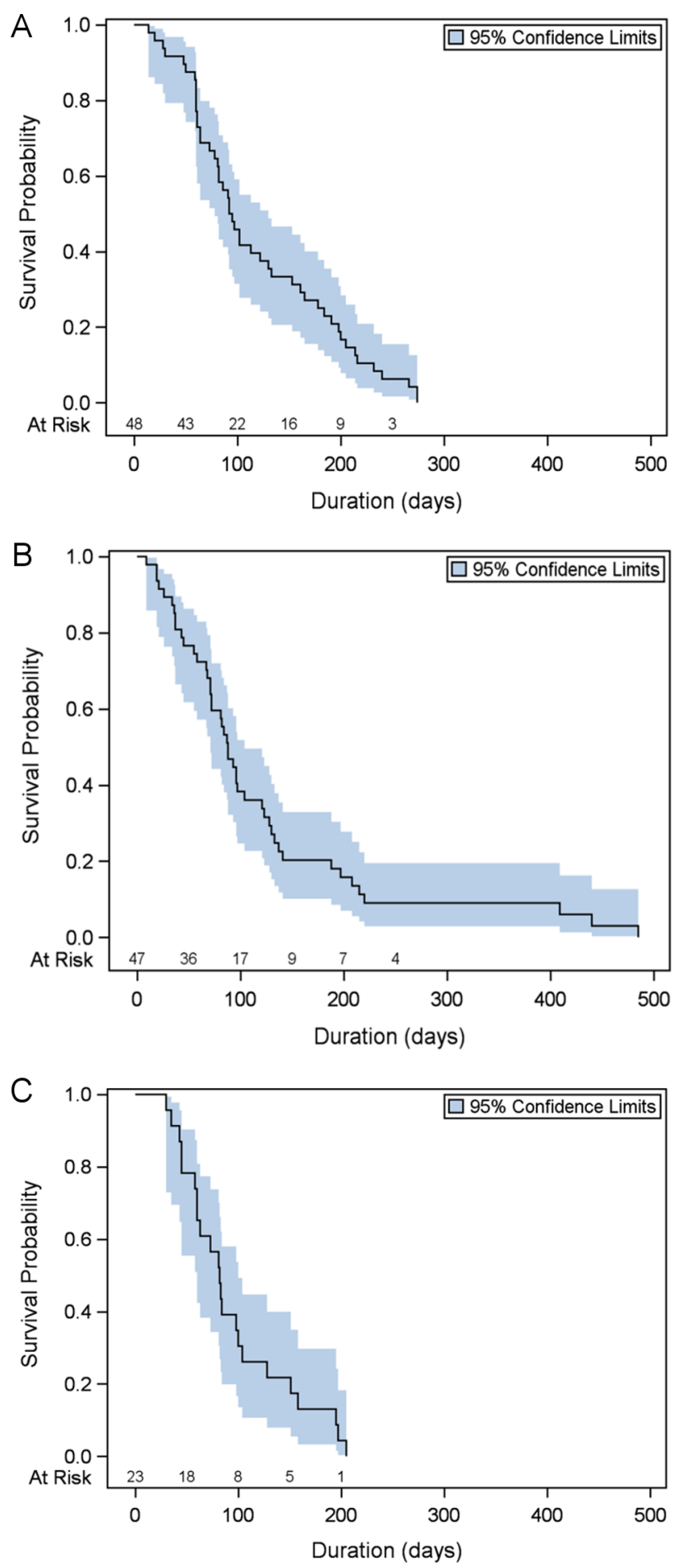

Figure 1. Kaplan-Meier estimates of progression free survival (PFS) in mCRC-patients treated before regorafenib defined as PFS1 (A), PFS2 (B), and PFS3 (C).

determined for Ras status impact, tumor localization, CEA or CA 19-9 level on the PFS under regorafenib (Ras: $\mathrm{HR}=0.75$ (95\% CI 0.39-1.43, $\mathrm{p}=0.375)$; localization: $\mathrm{HR}=0.71 \quad(95 \%$ 
Table 4. Progression free survival (PFS) of post-regorafenib treatment regimes and overall tumor response.

\begin{tabular}{lcc}
\hline Th post & $\mathbf{n}$ & \% \\
\hline Regorafenib & 28 & 100 \\
ECOG & & \\
0 & 19 & 66 \\
1 & 8 & 31 \\
2 & 1 & 3 \\
PFS all & median & quartiles \\
months & 2.7 & $2.0 ; 4.2$ \\
substance & $\mathrm{n}$ & $\%$ \\
Trifluridin/Tirpacil & 10 & 36 \\
PFS & median & quartiles \\
months & 2.7 & $2.0 ; 4.2$ \\
Chemo rechallenge & 18 & 64 \\
PFS & median & quartiles \\
months & 2.7 & $2.0 ; 4.9$ \\
response & $\mathrm{n}$ & $\%$ \\
CR & 0 & 0 \\
PR & 2 & 10 \\
SD & 4 & 19 \\
PD & 15 & 71 \\
ORR & 2 & 10 \\
DCR & 6 & 29 \\
\hline
\end{tabular}

CI 0.34-1.46, $\mathrm{p}=0.348) ; \log (\mathrm{CEA}): \mathrm{HR}=1.04$ (CI 0.91-1.19, $\mathrm{p}=0.595) ; \log (\mathrm{CA} 19-9): \mathrm{HR}=1.09$ (CI 0.96-1.23, $\mathrm{p}=0.184)$ ).

The proportion of patients with ongoing regorafenib treatment who did not progress or die within 3 months under regorafenib treatment was $48.7 \%$ (CI 32.5-63.2\%) of the 39 patients who had available PFS information. Of those, 19 were Ras wt 0: $68.4 \%$ (CI 42.8-84.4\%) and 20 were Ras mut 1,2: 30.0\% (CI 12.3-50.1\%). In addition, 56.3\% (CI 29.5-76.2\%) of patients with a CEA plasma level of $<=26.8 \mu \mathrm{g} / \mathrm{L} \quad(\mathrm{n}=16)$ did not progress or die and only $43.5 \%$ with higher CEA levels $(>26.8 \mu \mathrm{g} / \mathrm{L})$ did not suffer from progression or death. In addition, $61.5 \%$ of patients (CI 30.8-81.8\%) $(n=13)$ with a CA 19-9 level of $<=27 \mathrm{kU} / \mathrm{L}$ reached PFS over 3 month while only $42.3 \%$ (CI 23.5-60.0\%) in patients with higher CA 19-9 levels (>27 kU/L) did not suffer from progression or death.

Twenty eight patients with 2.7 months median PFS were treated following regorafenib therapy (quartiles 2.0; 4.2; Figure 1C). Of the 48 patient cohort, 20 (42\%) received no further treatment, 18 received chemotherapy re-challenge (median PFS 2.7 months) and 10 were treated with trifluridin/tripacil (median PFS 2.7 months). The disease control rate of trifluridin/tripacil was $4 \%$ and chemotherapy DCR was $18 \%$.

Toxicity. The most common adverse events in this regorafenib treatment were 24 (50\%) patients suffering fatigue and 21 (44\%) with hand-foot-skin reactions In addition, 9 adverse events were rated grade $3 ; 6$ patients $(13 \%)$ with hand-foot-skin reaction was the leading toxicity, plus one each with fatigue, diarrhea and mucositis/stomatitis. All other adverse effects including vertigo/nausea, hypertension, weight loss and hoarseness were rated mild at grade 1 or 2 .

\section{Discussion}

Our study is one of the first rare reports to assess the outcome of regorafenib therapy in refractory mCRC patients in a real-life setting. It includes stratification for tumor localization, Ras status and biomarkers such as CEA and CA19-9. Our data describes 48 patients $(29.2 \%$ women, $70.8 \%$ men; mean age 64.2 years) who were treated with regorafenib from 2013 to 2016 ; with $40 \%$ gaining disease control (4\% partial remission, $36 \%$ stable disease). The median PFS under regorafenib therapy was 2.9 months while PFS in the therapy directly given before was 3.1 month and 2.7 month in the therapy line following regorafenib application (Figure 1). The main adverse events at any grade were fatigue (in 50\%), hand-food skin reaction (in 44\%), hoarseness (in 40\%) and weight loss (in 27\%).

Only few real-life studies on experience with regorafenib treatment exist. In 2016, a group in the Ka-On Lam area of Hong Kong analyzed 45 patients with a mean age of 63 years which is comparable to this study (mean age 64 years) [11]. Median PFS was 3.9 months (95\% CI 3.3 to 4.5 months) and median OS was 7.6 month (95\% CI 4.2 to 11.1 months). Interestingly, in a comparative European study, survival data was also higher than that found in the original CORRECT trial [4]: in that 2017 Czech registry study, Kopeckova K. et al. [12] also report PFS of 3.5 months and median OS of 9.3 months in 148 patients; and these results are similar to our findings (PFS 2.9 months, Table 3, Figure 1B). Even if our data is retrospective, it remains consistent with other regorafenib studies [13]. Furthermore, PFS under regorafenib therapy (2.9 months) was quite similar with the PFS of therapy before regorafenib application (3.1 months) and also following it (2.7 months). Further studies, however, are required to evaluate therapy duration in later lines of treatment.

The median costs of patient management with regorafenib were calculated at 7,917€ per patient (10), and the biomarkers to predict beneficial treatment outcome must therefore be intensively investigated. Here we found that neither Ras status nor tumor localization (right, left) or biomarker levels of CEA or CA19_9, before treatment had any impact on PFS data. Furthermore, no correlation was found when testing for therapy response. Disease control rate (40\%) and overall response rate $(4 \%)$ were consistent with previous data from the original CORRECT trial [4] (ORR 1\%; DCR 41\%) and other retrospective evaluations from the Czech [12] (ORR 3\%; DCR 37\%) and Hong Kong cohorts [11] (ORR 3\%; DCR 36\%). When analyzing adverse events, fatigue (50\%) and hand-foot skin reaction (44\%) were most commonly observed. Grade III reactions of any symptom were not frequent and caused therapy dropout in only $2 \%$ of our patients. 
In September 2015, the Food and Drug Administration approved trifluridin/tripacil as further treatment options in $\mathrm{mCRC}$ after disease progression under standard therapy. Furthermore, re-challenge of chemotherapy following regorafenib application was suggested because regorafenib may act as a chemotherapy re-sensitizing agent $[14,15]$. However additional studies are needed to gain more information of the most beneficial therapy sequence for patients.

In this study, 28 patients (58\%) received further treatment following regorafenib therapy. Of these, $36 \%$ were treated with trifluridin/tripacil and $64 \%$ received chemotherapy $+/-$ target therapy. No difference in PFS (trifluridin/tripacil: 2.6 months; ChTh 2.7 months) was observed between trifluridin/ tripacil and chemotherapy $+/-$ targed therapy. This suggests that both options are possible, and retrospectively, the only difference was in disease control rate (trifluridin/tripacil 4\%; ChTh 18\%).

In conclusion, PFS and response under regorafenib treatment in our real-world setting were comparable to results from phase III clinical trials and other retrospective evaluations $[3,4,7,11,12]$. Moreover, Trifluridin/tripacil application or re-introduction of chemotherapy $+/$ - target therapy was effective following regorafenib therapy. Finally, prospective data is urged in order to gain more information on the most beneficial therapy sequence.

Acknowledgements: This work was supported by the Comprehensive Cancer Center Vienna at the Medical University of Vienna. Bayer Austria has supported this publication with an independent research grant. Bayer had no editorial control of this publication.

\section{References}

[1] FERLAY J, SOERJOMATARAM I, DIKSHIT R, ESER $S$, MATHERS $\mathrm{C}$ et al. Cancer incidence and mortality worldwide: sources, methods and major patterns in GLOBOCAN 2012. Int J Cancer 2015;136: E359-386. https://doi. org/10.1002/ijc.29210

[2] CHOI YJ, CHANG WJ, SHIN SW, PARK KH, KIM ST et al. The prognostic role of serum C-X-X chemokine receptor type 4 in patients with metastatic or recurrent colorectal cancer. Onco Targets Ther 2016; 9: 3307-3312. https://doi. org/10.2147/OTT.S104511

[3] VAN CUTSEM E, CERVANTES A, ADAM R, SOBRERO A, VAN KRIEKEN JH et al. ESMO consensus guidelines for the management of patients with metastatic colorectal cancer. Ann Oncol 2016; 27: 1386-1422. https://doi.org/10.1093/annonc/mdw235

[4] GROTHEY A, VAN CUTSEM E, SOBRERO A, SIENA S, FALCONE A et al. Regorafenib monotherapy for previously treated metastatic colorectal cancer (CORRECT): an international, multicentre, randomised, placebo-controlled, phase 3 trial. Lancet 2013; 381: 303-312. https://doi.org/10.1016/ S0140-6736(12)61900-X
[5] REY JB, LAUNAY-VACHER V, TOURNIGAND C. Regorafenib as a single-agent in the treatment of patients with gastrointestinal tumors: an overview for pharmacists. Target Oncol 2015; 10: 199-213. https://doi.org/10.1007/s11523014-0333-x

[6] KAKIZAWA N, SUZUKI K, FUKUI T, TAKAYAMA Y, ICHIDA $\mathrm{K}$ et al. Clinical and molecular assessment of regorafenib monotherapy. Oncol Rep 2017; 37: 2506-2512. https://doi.org/10.3892/or.2017.5456

[7] LI J, QIN S, XU R, YAU TC, MA B et al. Regorafenib plus best supportive care versus placebo plus best supportive care in Asian patients with previously treated metastatic colorectal cancer (CONCUR): a randomised, double-blind, placebo-controlled, phase 3 trial. Lancet Oncol 2015; 16: 619-629. https://doi.org/10.1016/S1470-2045(15)70156-7

[8] POVEDA A, GARCIA DEL MURO X, LOPEZ-GUERRERO JA, CUBEDO R, MARTINEZ V et al. GEIS guidelines for gastrointestinal sarcomas (GIST). Cancer Treat Rev 2017; 55: 107-119. https://doi.org/10.1016/j.ctrv.2016.11.011

[9] BRUIX J, QIN S, MERLE P, GRANITO A, HUANG YH et al. Regorafenib for patients with hepatocellular carcinoma who progressed on sorafenib treatment (RESORCE): a randomised, double-blind, placebo-controlled, phase 3 trial. Lancet 2017; 389: 56-66. https://doi.org/10.1016/S01406736(16)32453-9

[10] KIMURA M, USAMI E, IWAI M, GO M, TERAMACHI $\mathrm{H}$ et al. Comparison of cost-effectiveness of regorafenib and trifluridine/tipiracil combination tablet for treating advanced and recurrent colorectal cancer. Mol Clin Oncol 2016; 5: 635-640. https://doi.org/10.3892/mco.2016.1020

[11] LAM KO, LEE KC, CHIU J, LEE VH, LEUNG R et al. The real-world use of regorafenib for metastatic colorectal cancer: multicentre analysis of treatment pattern and outcomes in Hong Kong. Postgrad Med J 2017; 93: 395-400. https:// doi.org/10.1136/postgradmedj-2016-134547

[12] KOPECKOVA K, BUCHLER T, BORTLICEK Z, HEJDUK $\mathrm{K}$, CHLOUPKOVA $\mathrm{R}$ et al. Regorafenib in the Real-Life Clinical Practice: Data from the Czech Registry. Target Oncol 2017; 12: 89-95. https://doi.org/10.1007/s11523-016-0458-1

[13] CALCAGNO F, LENOBLE S, LAKKIS Z, NGUYEN T, LIMAT S et al. Efficacy, Safety and Cost of Regorafenib in Patients with Metastatic Colorectal Cancer in French Clinical Practice. Clin Med Insights Oncol 2016; 10: 59-66. https:// doi.org/10.4137/CMO.S38335

[14] BERTOCCHI P, AROLDI F, PROCHILO T, MERIGGI F, BERETTA GD et al. Chemotherapy rechallenge after regorafenib treatment in metastatic colorectal cancer: still hope after the last hope? J Chemother 2017; 29: 102-105. https:// doi.org/10.1080/1120009X.2016.1247205

[15] AVALLONE A, PICCIRILLO MC, ALOJ L, NASTI G, DELRIO $P$ et al. A randomized phase 3 study on the optimization of the combination of bevacizumab with FOLFOX/OXXEL in the treatment of patients with metastatic colorectal cancer-OBELICS (Optimization of Bevacizumab schedulIng within Chemotherapy Scheme). BMC Cancer 2016; 16: 69. https://doi.org/10.1186/s12885-016-2102-y 